\title{
OBJECTIVE STANDARDIZED CLINICAL ASSESSMENT WITH FEEDBACK: ADAPTING THE OBJECTIVE STRUCTURED CLINICAL EXAMINATION FOR POSTGRADUATE PSYCHIATRY TRAINING IN INDIA
}

PRABHA S. CHANDRA, SANTOSH K. CHATURVEDI, GEETHA DESAI

\section{ABSTRACT}

BACKGROUND: The objective structured clinical examination (OSCE) is used commonly for assessment of psychiatry trainees but has been used less for teaching. AIM AND SETING: This study describes the adaptation of the OSCE method for training purposes in a postgraduate psychiatry training center in India and the initial findings with regard to trainee performance. MATERIALS AND METHODS: The adapted method was called the objective structured clinical assessment with feedback (OSCAF). The adaptation included several steps - modifying existing OSCE patterns for language and cultural appropriateness, using supervised roleplaying instead of standardized or simulated patients and evolving an assessment method (14-item checklist) that would generate feedback. This exercise was conducted in front of a group of multidisciplinary peers and supervisors. STATISTICAL ANALYSIS: Descriptive statistics and ANOVA were used. RESULTS: Analysis of the performance based on the 14-item checklist assessing common elements of any patient-related interaction in 34 different OSCAF tasks indicated the following: less-than-satisfactory (<75\%) performances in the following areas - assuring confidentiality (73.5\%), assessing comfort (62\%), summarizing (60\%), closure $(62 \%)$ and checking whether the 'patient' had understood what was being communicated (42\%). Based on the nature of clinical situations, performances on some specific and difficult OSCAF situations were found to be inadequate and indicated need for further training. CONCLUSIONS: We have been able to demonstrate OSCAF to be a convenient, costeffective training method in psychiatry, with limited demands on resources; however, further refinements in ratings and feedback methods and studies on effectiveness may enhance its utility.

Key words: Medical education, objective structured clinical examination, postgraduate psychiatry, training

DOI: 10.4103/0019-5359.53391

Department of Psychiatry, National Institute of Mental Health and Neurosciences, Bangalore, India

\section{Correspondence:}

Dr. Prabha S. Chandra,

National Institute of Mental Health and Neurosciences,

Bangalore, India

E-mail: prabhasch@gmail.com

\section{INTRODUCTION}

The objective structured clinical examination (OSCE) format has been conventionally used for assessing medical students in various undergraduate and postgraduate 
settings. ${ }^{[1]}$ OSCEs have also been used in several specialties, including surgery, medicine, pediatrics and rehabilitation. ${ }^{[2-6]}$ However, there are only a few reports about using them for training purposes.

Brazeau et al. ${ }^{[7]}$ used the OSCE as a teaching tool following a request from the faculty and students, who wanted more direct observation and feedback on performance in various clinical scenarios. Following the use of the OSCE format for training, they concluded that the OSCE as a teaching tool was an efficient use of teaching resources. In addition, there was positive feedback from students about the opportunity to have constructive discussions on their strengths and weaknesses in clinical encounters and observe a variety of doctor-patient interaction styles, in addition to practicing for future OSCE-type examinations. Faculty members also reportedly enjoyed this teaching format and found the process of students giving feedback to their peers educationally useful. The teaching using OSCE was also extremely well rated in the end-ofrotation evaluations.

The OSCE as a training tool in the above exercise was adapted from the classic OSCE format and aimed at assessing the trainees and providing immediate feedback on various components of a clinical interview. Kligler et al. ${ }^{[8]}$ used competency-based directobservation tools in training family medicine residents to use an integrated medicine model in communication, and found the directobservation checklist to be a useful tool for feedback. Parish et al. ${ }^{[9]}$ used a five-station OSCE for training with different substanceuse disorders and found that the perceived educational value of the OSCE with feedback as a training tool was high, and feedback improved subsequent performance.

In psychiatry, while there are reports about the use of OSCEs in postgraduate examinations, their use in training has not been discussed even though several studies have reported that feedback after the examinations using the OSCEs is beneficial. In an opinion survey, Hodges et al. ${ }^{[10]}$ reported that Canadian psychiatry residents were uncomfortable about using OSCEs for examinations but felt that they would like the experience of using OSCEs as a part of their training.

In India, OSCEs are not commonly used as part of postgraduate evaluations though these have been used in undergraduate examinations. At the end of the 3 years of postgraduation training, trainees are examined by the conventional long and short cases in psychiatry, based on recommendations by the Medical Council of India. ${ }^{[11]}$ OSCEs have not yet been introduced in the examinations for postgraduate psychiatry.

The current paper describes an adaptation of the OSCE method for training purposes in postgraduate psychiatry in India. This paper focuses on two aspects - the methodology of adaptation; and an analysis of the performance of trainees in different dimensions of the clinical tasks based on 34 case scenarios.

\section{MATERIALS AND METHODS}

\section{Setting}

The institution where the current study was done is a premier postgraduate training 
center for psychiatry in India. Every year 20 postgraduate trainees enter the psychiatry residency program. While OSCEs are not used for examinations, as mentioned above, several faculty members of this institute, including the first two authors of this paper, have been trained in OSCEs as used in the UK for the MRCPsych.

It was felt that the OSCEs if adapted for use in training would be of considerable benefit in improving communication and assessment skills of trainees in the Indian setting.

\section{Adaptation of the OSCE format as OSCAF for training}

Several changes had to be made for use of the OSCE format as OSCAF (objective structured clinical assessment and feedback) for training purposes and for it to be culturally relevant. This included, firstly, the adaptation of various OSCE vignettes to the Indian setting, i.e., modifying content of the existing OSCEs used in the UK for MRCPsych examinations and adding a few components that were relevant to Indian psychiatric practice. For example, case scenarios such as 'discussing the illness with family members' were added because this is a frequently encountered situation.

The selection of clinical scenarios and adaptation was based on consensus between three psychiatric trainers. These clinical scenarios were then assessed for suitability in relation to content, timing and adaptability to roleplaying and used for the study. The case scenarios were chosen to cover a wide spectrum of subjects. Some of them included assessment of psychopathology (depression, thought disorder, suicidal ideation, insight); cognitive function assessments; different clinical conditions, such as substance use, eating disorders, sleep disorders; and different communication and interviewing challenges (education about Clozapine, educating a family member about schizophrenia, discussing compliance, breaking bad news). The case scenarios were based on commonly encountered situations in the inpatient, outpatient and emergency settings. Some OSCEs, such as assessing sexual problems, were not selected due to cultural issues about roleplaying certain topics in front of a peer group.

\section{Conducting training}

Postgraduate trainees from clinical psychology, psychiatric social work, psychiatric nursing, medicine and neurology are posted for 3 months in each adult psychiatry clinical unit. Objective structured clinical assessment and feedback (OSCAF) was conducted on the teaching day, for an hour, on a weekly basis. There are between 12 to 15 trainees in each teaching session, in addition to 5 to 7 trainers. The scenarios conformed to the psychiatrytraining curriculum. Each OSCAF consisted of 15 minutes of interview and a 15-minute discussion by the group and feedback. We decided to call this exercise OSCAF because firstly, we had borrowed the concept from the OSCE format, and secondly, it was objective as the task and assessments were both standardized; it was also structured there was formal and standard assessment by the three members of the faculty; and it was accompanied by a standard format of feedback.

A total of 34 OSCAFs were used over a 6-month period for training of two batches of 
psychiatry and allied postgraduate trainees, i.e., trainees in clinical psychology and psychiatric social work. Each batch consisted of 10 trainees who were posted for 3 months each, hence 20 trainees were involved. Each trainee had the opportunity to participate in at least one OSCAF task, while 7 of the 20 trainees were able to participate in two sessions involving different tasks. Trainees were given an introductory lecture on the OSCAF and the feedback methods at the beginning of the clinical posting.

\section{Role playing instead of simulated patients}

We introduced the use of role playing by trainees instead of standard or simulated patients because most institutions in India lack the resources to hire simulated patients. In order to overcome this, the trainees were trained in role playing based on standardized situations for each OSCAF. The trainee simulating the patient was given the scenario with specific written instructions about his/her role for different OSCAFs. Consent was taken from the trainee prior to the simulation. One faculty member briefed the trainees who were role playing the patient or family member about the simulation at least a few hours prior to the OSCAF. Following the OSCAF, debriefing was done to ensure comfort and for handling any difficulties related to the simulation. The debriefing included a session with both trainees that were part of the OSCAF situation, to check whether they felt uneasy or uncomfortable with the task, the assessment or feedback. Any possible emotional reactions were also assessed in some specific tasks that were difficult (such as the situation of breaking bad news).
Students that were evaluated for feedback on the OSCAF were informed at least a week earlier about the topic and situation so that they would be prepared.

\section{Assessment and feedback}

A form with 14 items assessing different aspects of the OSCAF scenario was devised. These 14 items [Table 1] were based on necessary aspects of any interview/ assessment/ interaction, irrespective of the subject. Of the 14 items, 3 assessed the content of the interview, while 11 items assessed the process. The same 14-item form was used to assess all 34 OSCAF scenarios. Based on the assessment of the specific situation, formal feedback was provided by three faculty members. A senior resident and peers constituting a multidisciplinary group (psychiatry, clinical psychology and psychiatric social work) were also asked to provide feedback. We were aware that performing in front of a group would be anxiety provoking and might influence performance. However, the group was briefed beforehand on the OSCAF method, and emphasis was laid on the fact that it was a training procedure and

Table 1: Percentages of satisfactory and unsatisfactory responses on 14 items

\begin{tabular}{lcc}
\hline Items rated & Unsatisfactory & Satisfactory \\
\hline Introduction & 3 & 97 \\
Assuring confidentiality & 27 & 73 \\
Taking consent & 6 & 94 \\
Stating objectives & 15 & 85 \\
Establishing rapport & 6 & 94 \\
Paraphrasing & 12 & 88 \\
Assessing comfort & 39 & 61 \\
Handling unexpected & 19 & 81 \\
situations & & \\
Checking for understanding & 57 & 43 \\
Summarizing & 37 & 63 \\
Closure & 29 & 71 \\
Completeness of task & 21 & 79 \\
Correctness of task & 18 & 82 \\
Clarity of task & 10 & 90 \\
\hline
\end{tabular}


constructive feedback would be appreciated. For example, the peer trainees were asked to first highlight the strengths of the clinical task performed and, instead of focusing only on the weaknesses, were asked to give suggestions about how the trainee could have improvised on the communication or intervention that was done in the OSCAF. Rules were laid down, which included confidentiality, no interruptions and positive feedback initially and constructive feedback at the end. Discussions and feedback were moderated by a faculty member.

\section{Assessments}

To establish uniformity of ratings, the three faculty members conducted a pilot to standardize ratings, agree on teaching points, and also discussed methods of providing feedback on all 14 measures. For every OSCAF, the three psychiatry faculty members rated each trainee on the 14 items on the checklist and gave their feedback based on that.

The 14 items in the checklist used for evaluating all 34 OSCAF situations were as follows: assuring confidentially, mutual introduction, obtaining consent, stating the objectives of the interview, establishing rapport, paraphrasing, assessing comfort, handling unexpected situations in the clinical scenario, checking for understanding, summarizing, closure, completeness of the task, clarity of communication and correctness of content. Each item was rated as satisfactory or unsatisfactory by the faculty members deputed for rating.

\section{Pilot to establish uniformity in assessment} ratings

A pilot of 15 OSCAF situations to standardize procedures and acquire training in assessment and feedback methods was done. To establish uniformity in the ratings on the 14-item checklist total scores of each OSCAF situation in the pilot and mean scores for each item were compared among the ratings done by the three faculty members. Comparison of the total scores and mean item scores between the three raters using ANOVA did not reveal any differences, indicating that the scoring was uniform between the three faculty members ( $F$ value, .723; sig, .579).

\section{Informed consent}

All trainees were informed that they would be rated on standardized measures by the faculty members. All identifying information was removed during the data entry process.

In addition, the trainees conducting the interview and the trainee doing the role playing were also given a feedback form to rate their subjective feelings and level of comfort felt during the OSCAF process. The data thus gathered, however, does not form part of this paper.

\section{Analysis}

The format of OSCAF in this study was designed to generate group-level data, mainly in order to identify curricular gaps (areas that are difficult for many residents), rather than assessment data for any single resident.

The following is a description of 34 OSCAFs conducted over a 6-month period for two batches of trainees (rotation of 3 months each).

We describe the results under two headings: satisfactory/ unsatisfactory ratings under each of 
the 14 items for all OSCAFs; differences in ratings on each item based on the nature of task in the OSCAF. For purposes of analysis, the OSCAFs were divided into two types of tasks: Task A included those mainly focusing on assessment and communication, and Task B included those providing information and education.

\section{OSCAFs classified as Task A}

The following are the OSCAFs classified as Task A: assessment of psychopathology (depression, thought disorder, delusions, suicidal ideation, insight); assessment of cognitive functions (frontal lobe, memory); assessment of pre-morbid personality, personal history, family history, suicidal risk, substance dependence; assessing different clinical conditions, such as alcohol use, eating disorders, sleep disorders, hypochondriasis, social phobia and chronic pain.

\section{OSCAFs classified as Task B}

The following are the OSCAFs classified as Task $\mathrm{B}$ : different communication and interviewing challenges (education about clozapine, educating a family member about schizophrenia, discussing compliance, breaking bad news, discussing side effects of antipsychotics, discussing behavior therapy, consent for electroconvulsive therapy, discussing tobacco use, grief, akathisia).

In all, there were 24 Task A and 10 Task B scenarios.

\section{RESULTS}

Comparison of scores between the three faculty members deputed for rating
Table 1 lists the percentages of unsatisfactory responses on the 34 OSCAFS on 11 items. As can be seen, most trainees (more than $85 \%$ ) performed satisfactorily on the following items: introduction, taking consent, establishing rapport, paraphrasing and clarity. The following items were rated as satisfactory less frequently $(75 \%-85 \%)$ : stating objectives, handling unexpected situations, completeness, correctness. The least number of satisfactory performances $(<75 \%)$ were seen in the following items: assuring confidentiality $(73.5 \%)$, assessing comfort $(62 \%)$, summarizing $(60 \%)$, closure $(62 \%)$ and checking whether the 'patient' had understood what was communicated (42\%).

The following difference in ratings was found based on the nature of tasks, i.e., Task A or Task B. More trainees performed poorly in Task $B$ on the following tasks: closure, assessing comfort, paraphrasing and confidentiality. In all other areas, the performances were equal.

A total score out of 14 was derived for each of the 34 tasks. Table 2 depicts the total number of satisfactory scores for each of the OSCAFs in rank order based on the topics.

Breaking bad news had the least satisfactory score, followed by assessing delusion, suicidal intent and family history. Assessing sleep disorder/ hypochondriasis/frontal lobe functions/ akathisia/ compliance, personal history and suitability for behavior therapy had the most satisfactory score.

The results from the debriefing sessions with the trainees indicated that they considered this as a useful learning experience. Some reported 
Table 2: Rank order of topics based on satisfactory scores

Topics

Total Score out of 14 based on

number of Satisfactory Responses

Breaking bad news

Assessing delusions

Taking a family history/ Assessing insight

Assessing suicidal intent

Discussion about starting Clozapine with a patient/Assessing personality

Assessing stream of thought/ Assessing for suitability of Acamprosate

Psychoeducation about schizophrenia/ Assessment of chronic pain/Discussing tobacco use

Assessing social phobia/ Establishing a diagnosis of alcohol dependence/Assessing akathisia/

Assessing eating disorders/ Assessing grief/ Assessing depression/ Taking informed consent for ECT 12

Taking a personal history/ Discussing behavior therapy for obsessive compulsive disorder

Assessing sleep disorder/ hypochondriasis/ frontal lobe functions/ executive functions /

akathisia/compliance; discussing side effects of antipsychotics

1

5

7

8

9

10

11

13

14 initial anxiety; however, they felt that the nature of feedback, which was nonjudgmental, specific and constructive, helped them. The trainees who simulated patients stated that they were able to empathize with their patients better after having been "in their shoes" for a short time. The faculty members felt satisfied with the objectivity of ratings and standardization of tasks, which helped them in giving constructive feedback. They also felt that more micro-level teaching was possible, and emphasis could be laid both on the process and content of the interview. However, different OSCAF situations may require different skills, apart from the 14 skills that were common to all situations. Not being able to assess more specific task-based issues was considered a limitation.

\section{DISCUSSION}

Our attempts at adapting the OSCE as an OSCAF for training purposes are still part of an ongoing process. However, based on our initial findings and experience, we feel that it can be a useful tool for training. The OSCAF as we have used it has several advantages. We are able to cover a number of topics so that during a 3-month rotation in the unit, on an average, a trainee is exposed to at least 12 case scenarios.
It also gives us the advantage to assess both the technical aspects, i.e., content; and factors related to the process, i.e., communication styles. By including tasks related to both assessment and education, we are able to focus on different aspects of doctor-patient interaction.

Using the OSCAF for training with both peer and supervisor feedback, is a useful process because it helps other trainees to think and modify their own styles based on their observations. Conducting the OSCAF in front of a multidisciplinary group comprising of trainees and supervisors helps in bringing to light different perspectives of the case, which might not otherwise be available to trainees.

Findings from our preliminary analysis indicate that some areas in a clinical interview, such as summarizing, closure and checking patient's understanding, are still areas that need more training. In addition, the trainees showed major skill deficits in handling some of the topics, such as breaking bad news; and in assessment of areas such as insight. Also of some concern is the poor performance in the assessment of suicidal intent.

Classic psychiatric training does not include 
this format; however, trainees often have to face these situations (e.g., suicidal patients, situations of breaking bad news) in wards and emergencies. Using a two-pronged approach where both content and process can be observed in a simulated situation, as well as enabling giving of feedback, offers a unique opportunity for training.

In India, OSCEs are not used as an examination method for postgraduation, and examiners still rely on the long case and short case format. This often prevents trainees from getting trained in these short-lived encounters with patients, which are sometimes more challenging and are also very common in dayto-day psychiatric practice.

Some of the difficulties with our adaptation include the need to ensure that the role playing is done appropriately, adequate briefing and debriefing procedures are in place and the whole group understands concepts of meaningful and appropriate methods of feedback. Team confidentiality is also important. While some initial efforts are needed to be made by the faculty for standardizing the case formats and role playing settings and ensuring that the feedback form is designed appropriately, the procedure subsequently becomes sustainable because of the standardization and uniformity applicable to each trainee batch.

Another issue which needs attention is to have refinements in ratings to make them more specific to the tasks, in addition to the general 14-item ratings followed above, based on some guidelines offered by Hodges. ${ }^{[2]}$
We hope that with these refinements and further standardization of procedures, we will be able to study the effectiveness of this form of training over a 3-month period and assess which of the unsatisfactory domains become satisfactory. It is possible that while some facets may improve, certain others might need a different training format. The data here is not intended to confirm whether the trainees learned or not, for which a different study design would be needed.

Some of the limitations of the current study include the fact that the trainees were at different levels of training; it might be better to look at skill strengths and deficits at specific levels of training. Also, though all the OSCAF situations have common process issues that trainees need to learn and have been rated by the 14-item checklist, there may be specific issues to each situation (such as suicide, depression, breaking bad news, i.e., content issues) that were not assessed. Finally, the study was carried out in a small group of trainees and with only three faculty members and has not been replicated. It needs to be conducted in larger groups of trainees, with more faculty members; and its utility in improving trainee performance needs to be studied before we can comment on its shortand long-term effectiveness.

\section{CONCLUSION}

The study has been able to demonstrate successful adaptation of the OSCE to the training arena by amalgamating it with assessment and feedback - coining a new term for it, viz., objective standardized clinical assessment with feedback (OSCAF), which is a 
convenient, cost-effective training method with limited demands on resources.

\section{ACKNOWLEDGMENTS}

We gratefully acknowledge the assistance given by Dr. Raghunandan in compiling the data and Ms. Roopa in data entry.

\section{REFERENCES}

1. Harden RM, Gleason FA. Assessment of clinical competence using an Objective structured clinical examination. Med Educ 1979;13:41-7.

2. Sloan DA, Plymale MA, Donnelly MB, Schwartz RW, Edwards MJ, Bland KI. Enhancing the clinical skills of surgical residents through structured cancer education. Ann Surg 2004;239:561-6.

3. Wilson BE. Performance-based assessment of internal medicine interns: Evaluation of baseline clinical and communication skills. Acad Med 2002;77:1158.

4. Hodder RV, Rivington RN, Calcutt LE, Hart IR. The effectiveness of immediate feedback during the objective structured clinical examination. J Med Education 1989;23:184-8.

5. Hilliard RI, Tallet SE.The use of an objective structured clinical examination with postgraduate residents in pediatrics. Arch Pediatr Adolesc Med 1998;152:74-8.

6. Jain SS, Nadler S, Eyles M, Kirshblum S, DeLisa
JA, Smith A. Development of an objective structured clinical examination for physical medicine and rehabilitation residents. Am J Phys Med Rehab 1997;76:102-6.

7. Brazeau C, Boyd L, Crosson J. Changing an existing OSCE to a teaching tool: The making of a teaching OSCE. Acad Med 2002;77:932.

8. Kligler B, Koithan M, Maizes V, Hayes M, Schneider C, Lebensohn P. Competency-based evaluation tools for integrative medicine training in family medicine residency: A pilot study.BMC Medical Education 2007;18:7-7.

9. Parish SJ, Ramaswamy M, Stein MR, Kachur EK, Arnsten JH. Teaching about Substance Abuse with Objective Structured Clinical Exams. J Gen Intern Med 2006;21:453-9.

10. Hodges B, Hanson M, Mcnaughton N, Regehr G. What do psychiatry residents think of an Objective Structured Clinical Examination? Academic Psychiatry 1999;23:198-204.

11. Medical Council of India. Postgraduate medical education regulations, 2000. Available from: http://www.mciindia.org/know/rules/rules_pg.htm. [last retrieved on 2008 Mar 14].

12. Hodges B, Hanson M, McNaughton N, Regehr G. Creating, monitoring, and improving a psychiatry OSCE: A guide for faculty. Academic Psychiatry 2002;26:134-61.

Source of Support: Nil, Conflict of Interest: None declared. 